\title{
MORPHODYNAMIC PROCESSES IN ESTUARIES - COMPARISON OF MARINE AND LIMNIC TIDAL FLATS
}

\begin{abstract}
Thorsten Albers ${ }^{1}$, Dagmar Much $^{2}$, Nino Ohle ${ }^{2}$, Nicole von Lieberman ${ }^{1}$, Eva Falke ${ }^{1}$
Tidal flat areas in estuaries are affected by strong morphodynamics. Changes of sedimentation and erosion occur on different temporal and spatial scales. These changes challenge the responsible authorities due to the high importance of sufficient navigation channel depths and the ecological importance of those unique zones. In cooperation with the Hamburg Port Authority the Hamburg University of Technology runs broad field measurements on tidal flat areas in the Elbe estuary. The results provide a fundamental data set to improve the knowledge about morphodynamic processes. For more than 3 years water levels, waves, current parameters and suspended sediment concentrations are being recorded continuously and in a high resolution at different positions on a marine investigation area. Therefore, ADCP's, optical backscatter sensors (OBS) and pressure transducers (PT) are used. To observe the consequences of the morphodynamic processes, the bathymetry of the investigation areas is determined with a multi-beam echo sounder (MBES) in frequent intervals as well as after extreme events like storm surges. Derived from the field data certain patterns of erosion, sediment transport and sedimentation could be observed depending on tidal currents, waves and large scale weather conditions. Seasonal effects are analyzed as well as the influence of extreme events. The observed processes are compared with data from a limnic investigation area, where a second, shorter field study was carried out.
\end{abstract}

Keywords: Tidal Flats, Field Measurements, Morphodynamics, Sediment Transport

\section{INTRODUCTION}

The North Sea is an epeiric sea of the Atlantic Ocean. Thus, the oscillation of the Atlantic affects the North Sea from the north and through the British Channel. Huge tidal flat areas, long tidal channels and creeks, ripples and dunes have an effect on the view of the German North Sea coast. Especially the areas of the Wadden Sea underlie sediment relocations on different scales.

The most important waterways at the German coast are the estuaries Elbe, Weser and Ems. In spite of several measures, that stabilize the waterways, strong morphodynamic changes occur. Especially in the mouth of the estuary Elbe these permanent displacements challenge the stakeholders and decisionmakers. The Elbe is the approach to the port of Hamburg and thus it may be called a lifeline of a whole region. The port of Hamburg is a turntable of the international container shipping, the largest port in Germany and the second largest in Europe. To live up to expectations of many different parties a holistic and innovative estuary management is necessary.

A broad process-knowledge is necessary to assess the resulting risks and to find an agreement between utilization demand and ecological meaning of the affected coastal zone. Due to its high sensitivity against hydro- and morphodynamic changes tidal flat areas, especially in the mouths of estuaries, move over to the center of scientific activities. Big challenges in this interrelation are the impacts of the climate change.

The aim of the research project discussed in this paper is the analysis of morphodynamic and hydrodynamic changes in the investigation area "Neufelder Watt" in the mouth of the estuary Elbe. Supported by the Hamburg Port Authority (HPA) the Institute of River and Coastal Engineering of the Hamburg University of Technology runs extensive field measurements to improve the process knowledge on tidal flats. The results provide a secure theoretical background for the mathematical multi-dimensional modeling of morphodynamic processes on tidal flats. The comparability of morphodynamic processes on marine and limnic tidal flats is verified by measurement campaigns on a second investigation area near the port of Hamburg.

\section{INVESTIGATION AREAS}

The field measurements focus on two investigation areas, which are shown in figure 1: One area is located in the mouth of the estuary Elbe and represents a marine tidal flat. The second investigation area is located near the city of Hamburg and typifies a limnic tidal flat area.

\footnotetext{
${ }^{1}$ Institute of River and Coastal Engineering, Hamburg University of Technology, Denickestr. 22, 21073 Hamburg, Germany

${ }^{2}$ Hamburg Port Authority, Hydrology, Neuer Wandrahm 4, 20457 Hamburg, Germany
} 
The main field investigations take place in the Neufelder Watt with the "Neufelder Sand" in the mouth of the estuary Elbe (figure 2). The area under investigation is in close interaction with the main stream of the Elbe. The average tidal range in the investigation area is about 3 meters. The Wadden Sea areas around the "Neufelder Rinne" fall dry during a longer period around low tide. The water depths during an average tidal high water amount to $1 \mathrm{~m}$ to $1.5 \mathrm{~m}$ northwest of the channel and $1.5 \mathrm{~m}$ to $2 \mathrm{~m}$ southeast of the channel. The largest water depths in the main part of the channel add up to $4.5 \mathrm{~m}$ at mean high tide according to measurements in September 2008. The inlet was silted up highly at that time so that the water depths in that area are only a few centimeters at mean tidal low water. The sediments in that investigation area mainly consist of fine sands with a medium grain size of $0.11 \mathrm{~mm}$.

Figure 2 shows the bathymetry in the mouth of the estuary Elbe with the Wadden Sea areas and the "Medemgrund" as the most important shallowness. The picture detail shows the focus area.

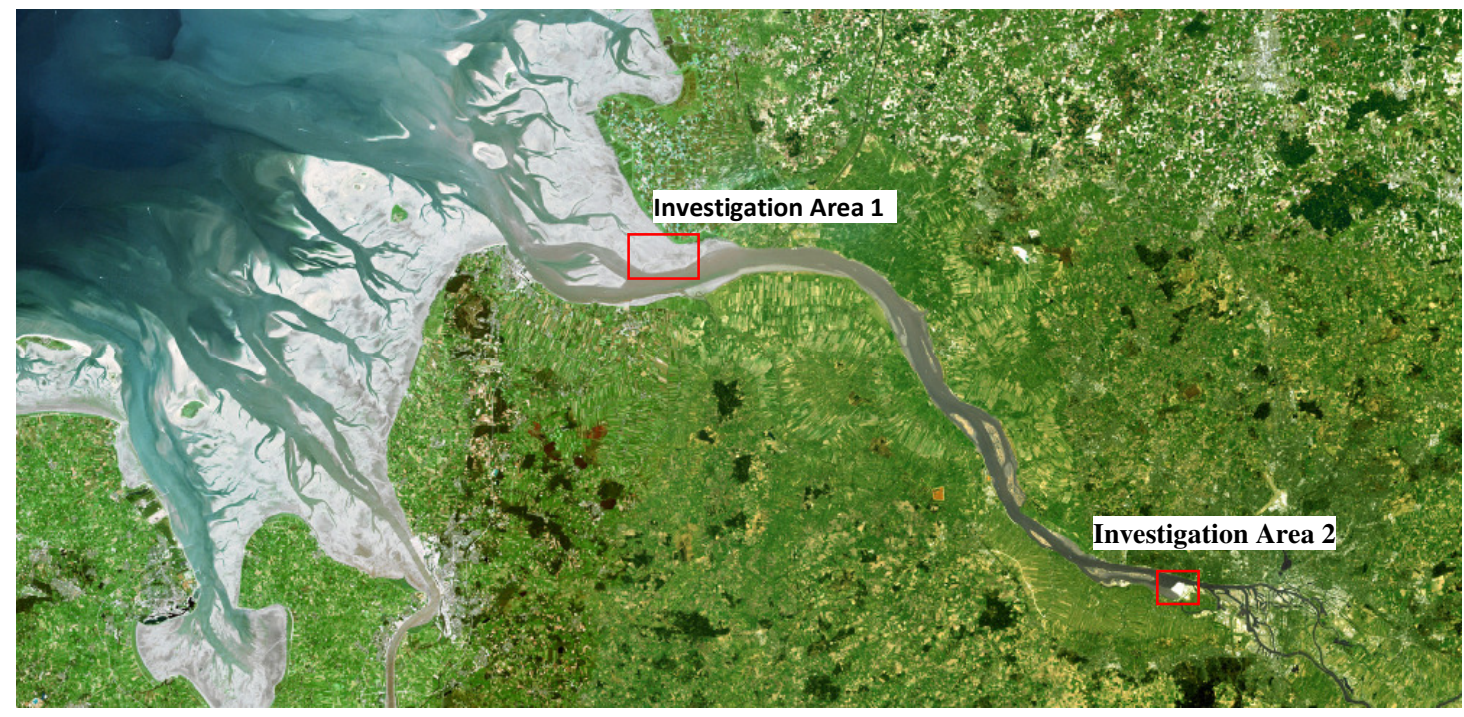

Figure 1. Map of the Elbe estuary with marked investigation areas (Image Editing, Copyrights: Brockmann Consult, Common Wadden Sea Secretariat (c) 2003).

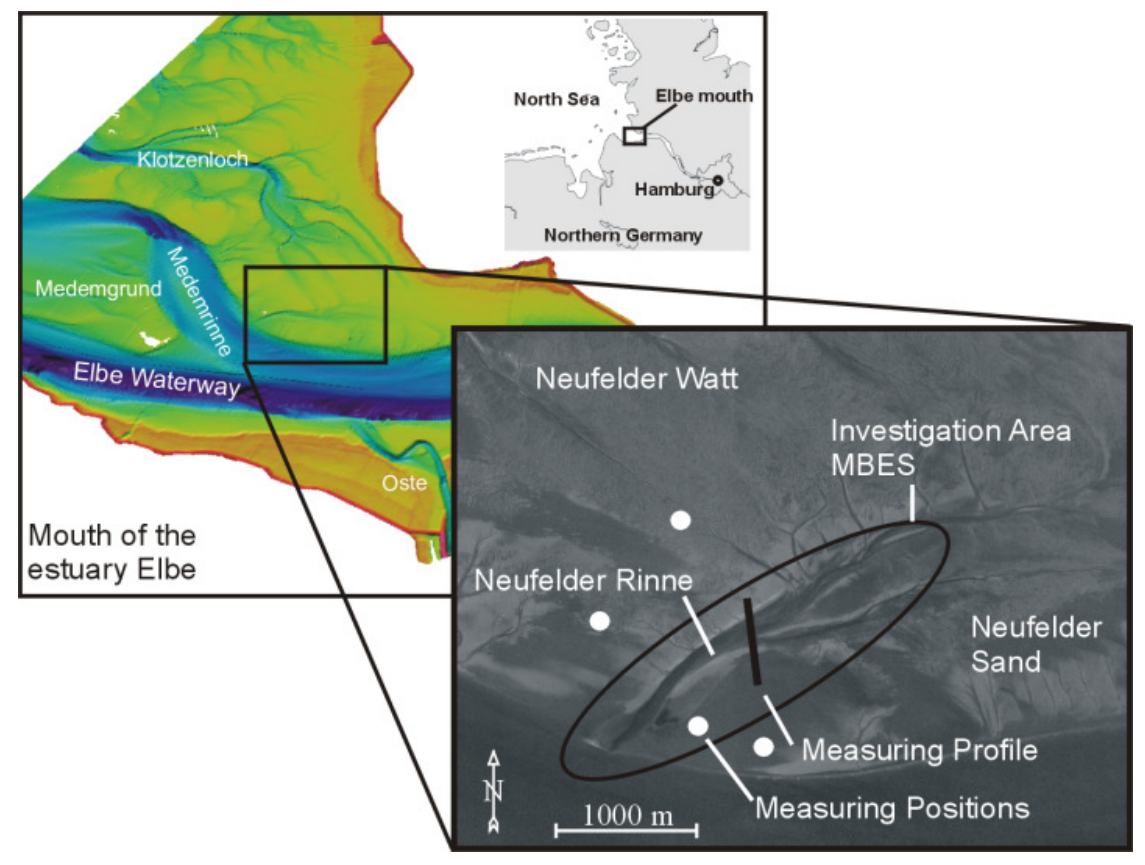

Figure 2. Investigation area and measurement positions in the mouth of the estuary Elbe. 


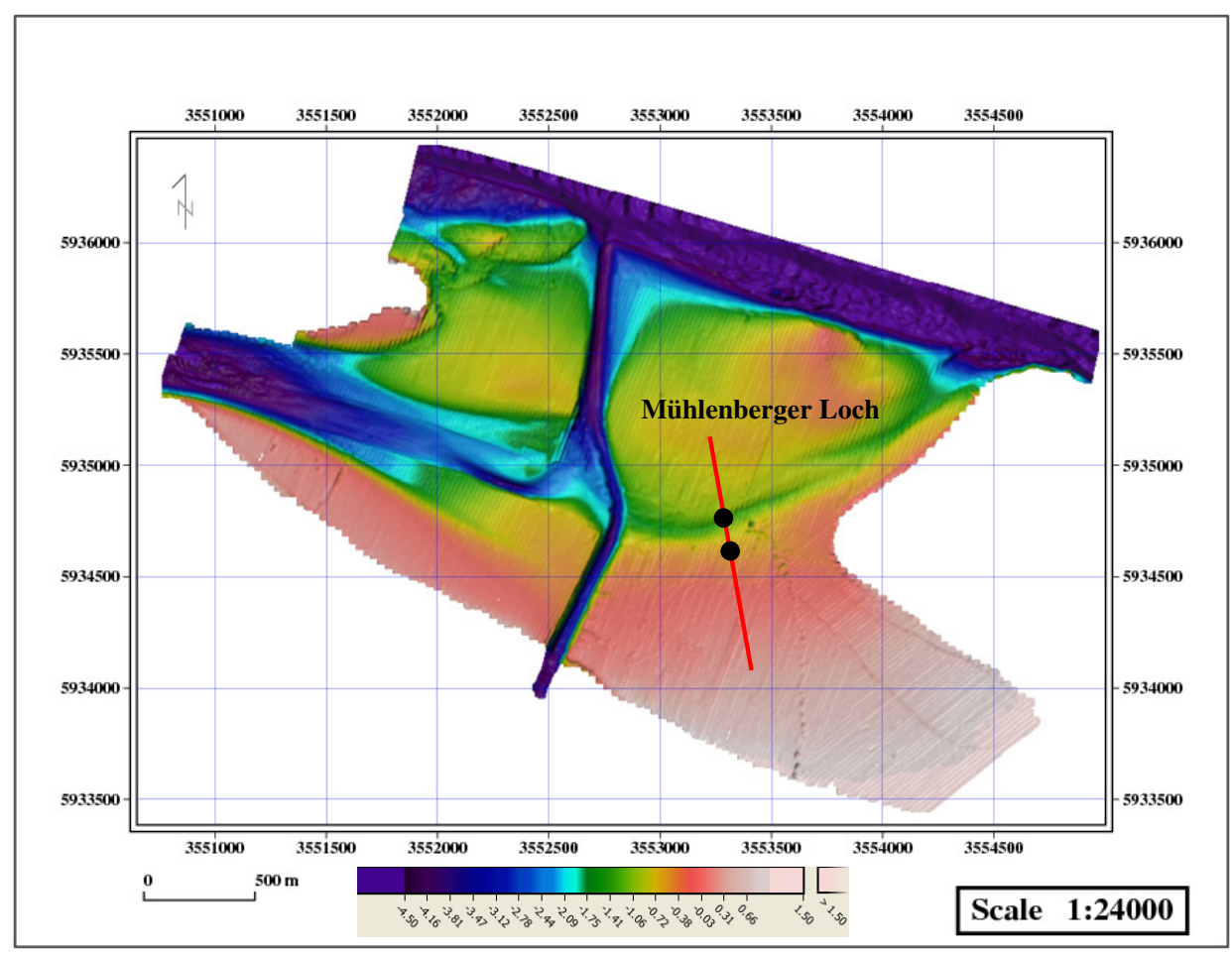

Figure 3. Limnic investigation area near the city of Hamburg including measurement positions (black) and analyzed profile (red).

Figure 3 shows the second investigation area "Mühlenberger Loch" near the port of Hamburg. The field investigations concentrate on the creek that intersects the area. The morphologic changes are analyzed along the marked profile. The sediments in the limnic tidal flat area consist of silt with a medium grain size of $0.036 \mathrm{~mm}$.

\section{FIELD MEASUREMENTS}

In summer 2006 firstly a cross section in the Neufelder Rinne was selected to install the measurement devices (cf. figure 2). The equipment was positioned in the middle of the creek as well as on both banks. Flow parameters, sediment concentrations and waves were measured permanently in a high resolution. In the following phases of the project the measuring positions were installed at areas of higher elevation northwest and southeast of the channel. In the process three Acoustic Doppler Current Profilers (ADCP) were used as well as six pressure sensors and three Optical Backscatter Profilers, which allowed an assessment of the suspended sediment concentration (SSC). Additional to the permanent installed instruments multi-beam echo sounder (MBES) measurements were performed in the channel and on the surrounding areas in frequent intervals of three to five weeks and after extreme events. Furthermore, soil samples were taken. They were completed to a sediment register of the area under investigation.

The arrangement of the measuring devices in one position is shown in figure 4. Flow parameters were recorded continuously and in a high resolution with three RDI ADCP Workhorse Sentinel. Over a period of 5 minutes an ensemble of 50 pings was collected, whereas the accuracy of the flow velocity is $\pm 0.3 \mathrm{~cm} / \mathrm{s}$ and the one of the flow direction $\pm 2^{\circ}$. Suspended sediment concentrations were measured with three Argus Surface Meters (ASM) by ARGUS Environmental Instruments. In that innovative measuring instrument 100 optical backscatter sensors (OBS) mounted in a steal bar assessed the sediment concentration over a one-meter column above the sea bottom. Before the deployment the device was calibrated with the suspended matter occurring in the area under investigation. Every 5 minutes 5 samples were collected and averaged, whereas the accuracy is $\pm 10 \%$. Regularly suspended matter samples were taken and compared with the results of the ASM. Six pressure sensors recorded waves with a measurement frequency of $10 \mathrm{~Hz}$. Those sensors are a self-construction of the institute. 
Data can be collected self-sustainingly over a period up to three months and is stored on a MMC memory card. All other devices work autarkic as well.

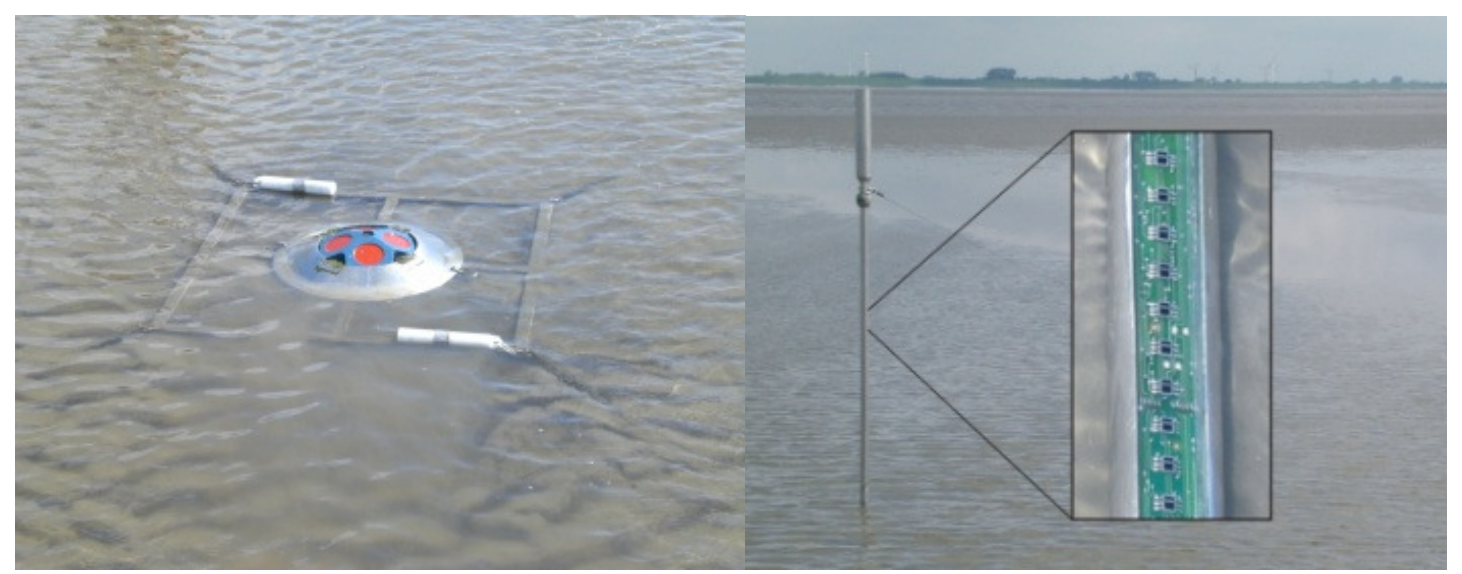

Figure 4. Measuring position with ADCP, PT's (left) and OBS device (right).

In regular intervals of three to five weeks the bathymetry of the marked investigation area was analyzed with a multi-beam echo sounder. Furthermore, measuring tours were attempted as soon as possible after extreme events. Therefore the research vessel "Nekton" of the Institute of River and Coastal Engineering comes into operation (Albers \& von Lieberman, 2007). To survey the bathymetry a built-in multi beam sonar Elac Nautik Seabeam 1185 with a frequency of $180 \mathrm{kHz}$ is used. It is especially appropriate for surveys in shallow water but also applicable for water depths up to $300 \mathrm{~m}$. The tide correction is done by Real Time Kinematics (RTK Tides) and the vessel's movements are compensated by an Octans gyrocompass.

In two measurement campaigns of four to six weeks in 2009 and 2010 all the parameters mentioned above were recorded at two positions in the limnic investigation area. The setup of the instruments was the same as in the marine area. Available bathymetric data sets were analyzed and complemented by additional multi-beam soundings.

\section{RESULTS}

\section{Marine investigation area}

Derived from the recorded current parameters and the suspended sediment concentrations the sediment transport rate for various positions in the marine investigation area was analysed and plotted together with wind parameters (figure 5). Extreme events may cause a short peak (08/05/08), but if their duration is limited, they do not lead to significant bathymetric changes. Depending on the wind direction even higher wind velocities do not result in a pronounced peak (09/13/08). Significantly larger sediment transport occurs during and after phases with longer-lasting high wind velocities from western directions. Seasonal variations of the transport rate are due to a larger viscosity of the water in winter resulting in smaller settling velocities of the suspended sediments.

From the recorded and analyzed sediment concentrations and the flow parameters the residual transport was calculated by balancing the transported material (figure 6).

On the tidal flats the sediment transport during flood phase overbalanced. Therefore, the sediment transport is directed to the east. If the exposure of the measurement position is larger, the amount of transported material increases. The main tidal creek Neufelder Rinne interrupts this eastward directed transport. Material deposits at the western slope and pushes the creek's axis to the east. Largest changes occur at the edge of the tidal flat area, where the transport is largest.

In the Neufelder Rinne the residual transport during ebb tide overbalanced. The suspended sediment is carried out of the creek and forms an ebb delta, which is clearly visible on the sonar soundings. 

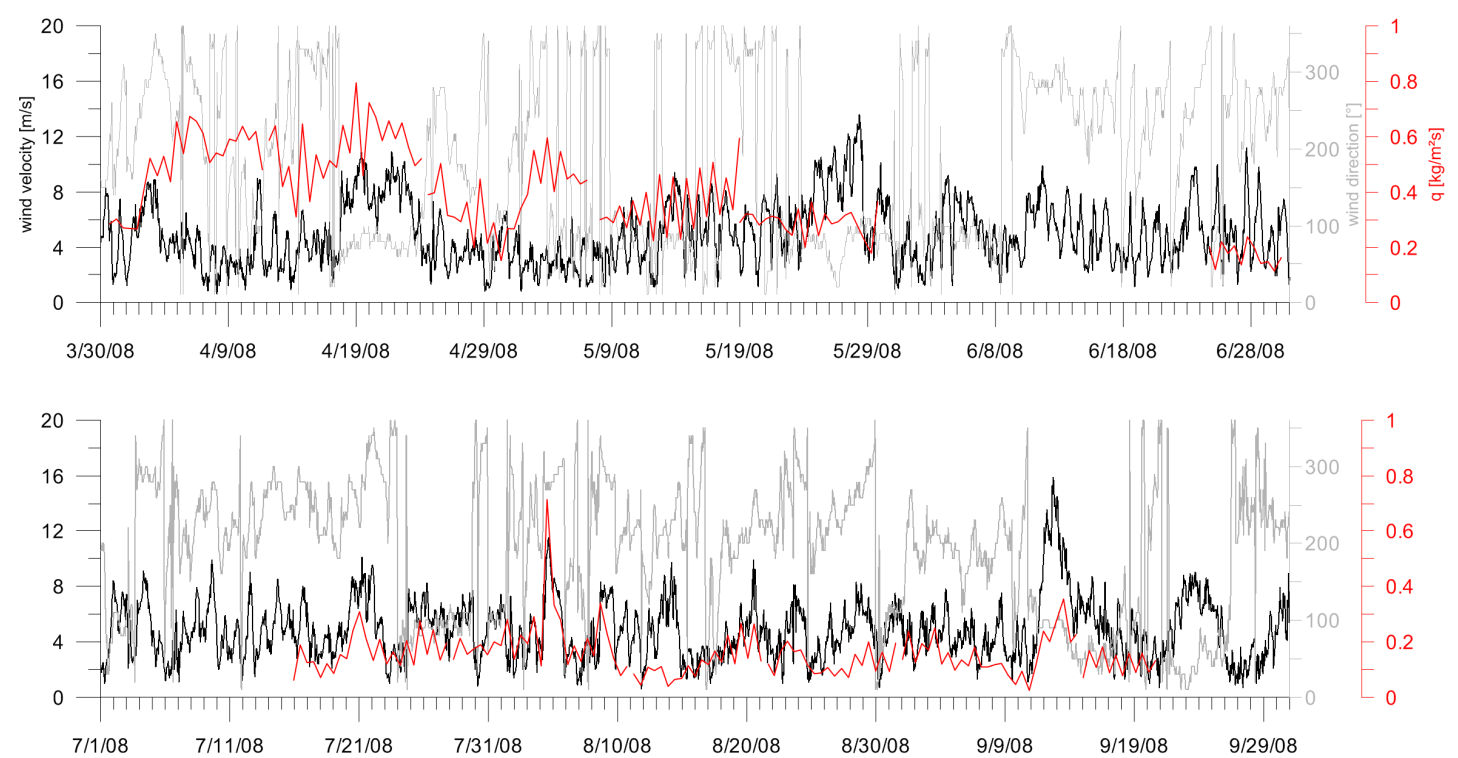

Figure 5. Sediment transport rate in $\mathrm{kg} / \mathrm{m}^{2} \mathrm{~s}$ (red), wind speed (in $\mathrm{m} / \mathrm{s}$; black) and wind direction (in degrees; grey) in the investigation area from July to September 2008.

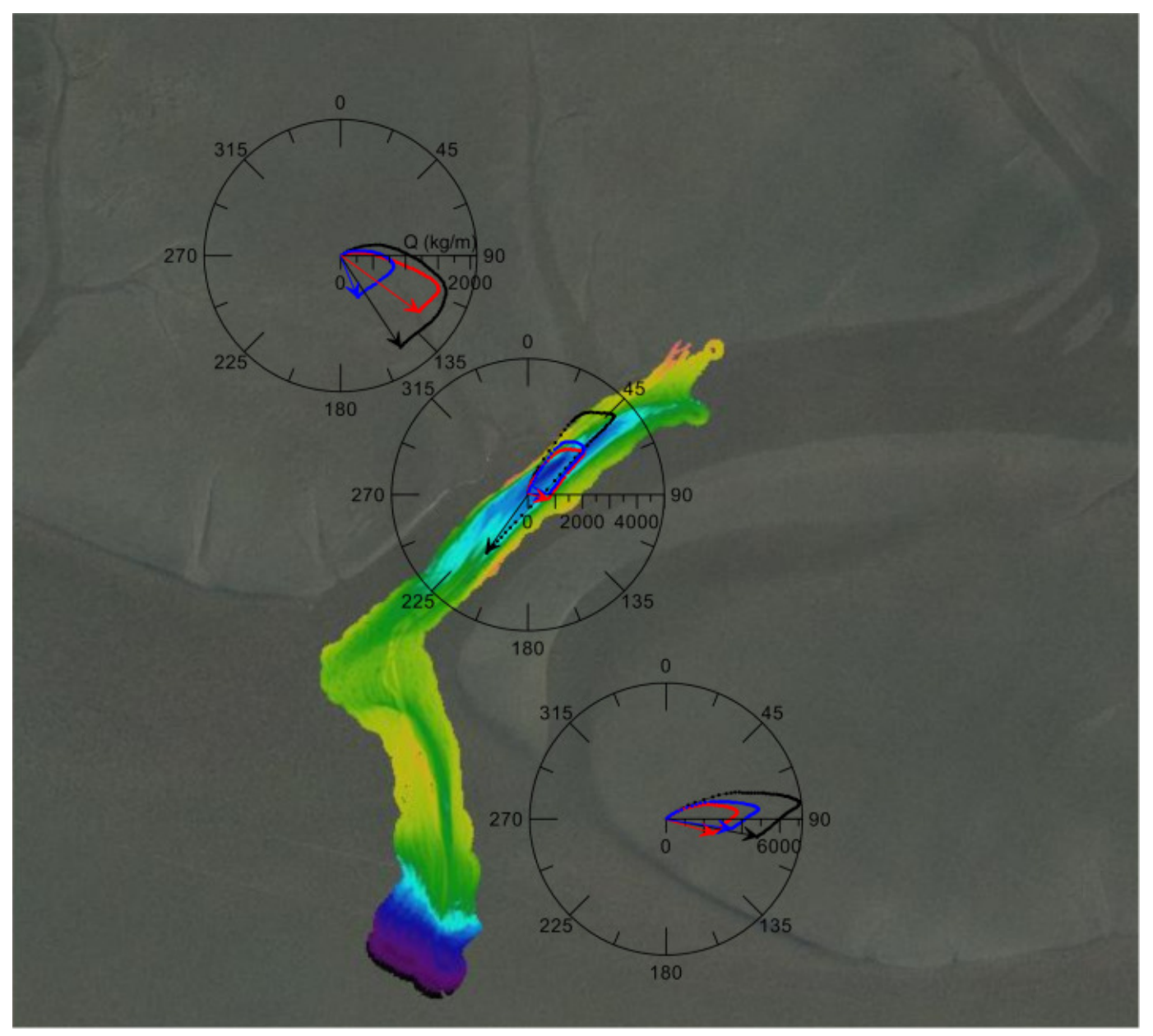

Figure 6. Residual sediment transport during various tides in the marine investigation area; bathymetry of September, $25^{\text {th }} 2008$. 
The results of the multi-beam echo soundings show a separation of the Neufelder Rinne into the highly dynamic part of the inlet and the more stabile but still continuously moving main part.

Figure 7 shows the relocation of the inlet of the Neufelder Rinne derived from the results of different multi-beam echo soundings. The strong dynamics are obvious. Essentially the inlet moves to the east, whereas the relocation can be up to one hundred meters per month. It continuous until an instable situation is reached (e.g. 06.05.2008). Then the ebb stream erodes the undercut slope of the main part of the creek and a new inlet is formed, which lies at a more western position. For a short while two inlets exist, until the older one is silted up. This reversible development is visible e.g. between April 23rd and August 21st 2008. Subsequent the process begins again. The bathymetry in figure 6 shows a situation on September $25^{\text {th }} 2008$ short before this procedure happens. On November, 6th 2008 when the multibeam soundings had to be interrupted due to the weather, the inlet was at a position similar to the one from August, 21st 2008. So between September and November again an instable situation was reached and the inlet shifted back. This primary oscillation with a period of several weeks was also visible in other years. The period of the oscillation varies over a year.

The horizontal displacement especially of smaller creeks was already mentioned by Ehlers (1988). Different observations showed that those creeks may change their positions about a few decimeters per tide. These changes are reversible and do not lead to large medium-term changes. Observations at the Neufelder Rinne confirm that.

At the northeast end of the main part of the Neufelder Rinne the creek runs into a west-east course which was very stable between 2006 and 2009. The main part underlay a continuous movement from northwest to southeast between 2006 and 2007. At the northwest bank sediment deposited and the slope shifted to the southeast. The southeast bank was eroded. The course of the main part changed from a southwest-northeast course to a steeper SSW-NNE course. In 2008 and 2009 the situation reversed and the main part shifted back to northwest. Also the course changes to a more southwest-northeast one like it was in 2006. This secondary oscillation with a period of years is also visible in figure 7 , where the range of the position of the inlet shifts from northwest to southeast and back.

The described relocations occurred both, during summer and winter months. Direct impacts of two recorded heavy storm surges on the bathymetry could not be observed.

On November 1st 2006 a storm surge with water levels of approximately 3 meters above mean high tide occurred. Minor changes near the inlet were not due to that extreme event but due to the continuous movements. On November 9th 2007 another storm surge also with water levels of approximately 3 meters above mean high tide appeared. Direct consequences of that event on the bathymetry were again not visible.

\section{Limnic investigation area}

During the first measurement campaigns in the limnic investigation area a first-cut analysis of the hydrodynamics and morphodynamics in that area was done. Besides the check, if the measurement setup that was used on the marine tidal flats is also applicable in the limnic surroundings, first information about the morphodynamic processes were gained.

In the investigation period effects of waves on the sediment concentration could not be detected. Due to the short duration of the first measurements only a few different weather conditions could be analyzed. Changes depending on single events or seasonal effects of course could not be considered. The course of the sediment concentration mainly depends on the flow velocities. The data of the different measuring positions show almost identical results. The peaks in the flow velocity during flood tide are directed to the southeast. They are factor 1.2 up to factor 2.0 larger as the peaks during ebb tide. According to this, the residual sediment transport is directed to the east, southeast respectively. At the eastern end of the creek sediments are deposited. The ebb current is not large enough to transport that material out of the creek. The results are a slow siltation of the creek from east to west and increasing heights of the areas south of the creek. These morphologic changes are illustrated in figure 8 , which shows the development of the profile marked in figure 3. The large differences between July 2002 and July 2003 are due to dredging of the creek. It was meant to increase the drainage of the area to avoid further siltation. The measure was not successful and the process of siltation started again directly after the dredging.

In the present situation, the influence of the creek on the hydrodynamics of the area is limited. Only in the beginning of the flood phase the currents follow the course of the creek. After a short time the creek is overflowed crosswise. Also during ebb tide the creek is overflowed crosswise for a long time. 
Not until the water level falls below the surrounding tidal flats, the current directions follow the course of the creek.
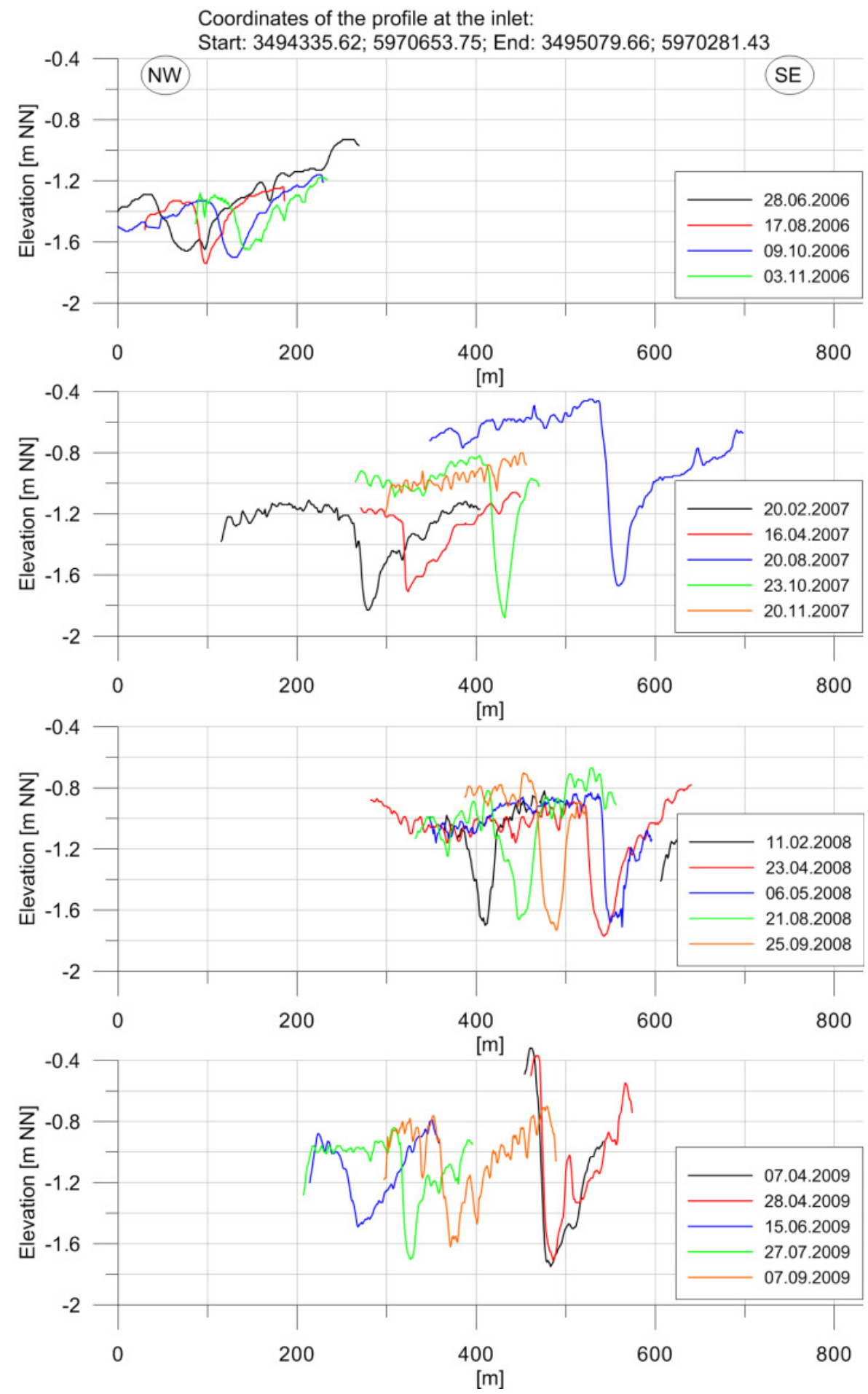

Figure 7. Relocation of the inlet of the main tidal creek in the investigation area. 

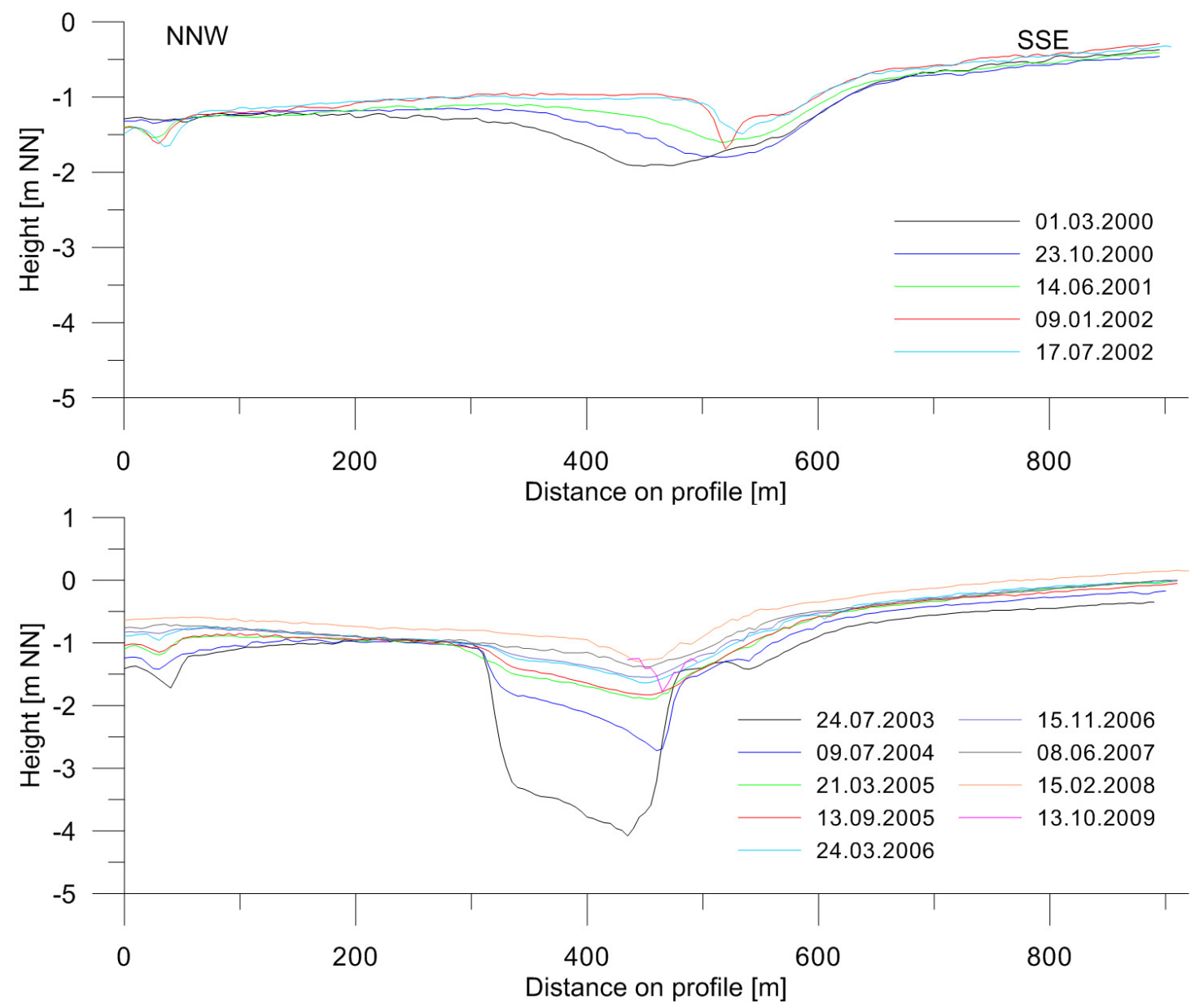

Figure 8. Morphologic changes of the profile in the limnic investigation area.

\section{CONCLUSIONS}

The eastward directed shift of the inlet due to the sediment transport continues until the angle between the main part of the creek and the inlet gets too acute. Then erosion at the undercut slope of the ebb stream dominates and a new inlet develops. After that the overall sediment transport dominates again. This development was visible through all the observation period.

The relocation of the main part of the Neufelder Rinne to the southeast between 2006 and 2009 could also be explained by the overall sediment transport. The course of the main part changed into a steeper SSW-NNE course. At a certain point the dominant ebb stream within the Neufelder Rinne erodes the northwest bank and the creek's axis shift back to northwest.

Both described oscillations pass off continuously during the whole year. Because of the shorter period of the oscillation of the inlet, it is a good indicator for morphological activity. The relocation is accelerated at certain times. Larger sediment transport rates with a longer duration accelerate the relocation. This is indicated in March and April 2009 (cf. figure 5) when the relocation reaches large rates (cf. figure 7). Single extreme events of short durations only increase the transport rate for a few days. This does not have significant influences on the bathymetry.

The residual transport in the limnic investigation area is directed to the east and southeast respectively. This leads to siltation of the creek, which is overflowed crosswise, and the tidal flat areas south of it. The ebb stream through the creek is not large enough to resuspend deposited material and transport it out of the creek. Consequently the ebb stream on the tidal flats south of the creek decreases and also here deposited material is kept in place. During the observed periods, the processes were continuous. Due to the investigation duration, conclusions about seasonal influences or effects of extreme events could not be drawn. 


\section{SUMMARY}

Facing the high importance the free approach to the port of Hamburg means to the economic development of Northern Germany, a sustainable estuary management is required. In particular the knowledge about morphodynamic processes on tidal flats needs to be improved.

On the basis of extensive high-resolution field investigations the Institute of River and Coastal Engineering at the Hamburg University of Technology analyzed morphodynamic processes in a tidal flat area in the mouth of the estuary Elbe. It is the intention to improve the process knowledge of sediment transport on tidal flats.

From the measurements at various positions, the residual sediment transport was computed and the process of the overall transport patters could be derived. Morphodynamic tendencies and relocations were recorded in the creek Neufelder Rinne and the nearer surroundings with a multi-beam echo sounder. The transport patterns, measured sediment transport rates and the recorded morphological changes could be correlated.

The transferability of the processes observed and analyzed in the marine environment to the limnic tidal flat area was verified with two measurement campaigns in the second investigation area "Mühlenberger Loch". Based on available bathymetric data, the siltation of the creek and the increasing heights of the area south of the creek were shown. During the measurement periods the same setup as in the marine environment was applied. The field data show that suspended sediments are mainly transported to east and southeast. Due to low ebb currents this leads to siltation of the creek and the areas south of the creek.

\section{REFERENCES}

Albers, T., von Lieberman, N., 2007: Monitoring of hydrodynamics and morphodynamics in a tidal flat area. In: Penchev, V. and Verhagen, J.-H. (Eds.): Proceedings of the Fourth International Conference on Port Development and Coastal Engineering, Varna, Bulgaria, ISBN 978-95492103-1-6, Black Sea Coastal Association, Bulgaria.

Ehlers, J., 1988: The Morphodynamics of the Wadden Sea. Balkema. Rotterdam, The Netherlands. 